\title{
Deterministic Performance Bounds on the Mean Square Error for Near Field Source Localization
}

\author{
Mohammed Nabil EL KORSO, Student Member, IEEE, Alexandre RENAUX, Member, IEEE, Rémy \\ BOYER, Member, IEEE and Sylvie MARCOS
}

\begin{abstract}
This correspondence investigates lower bounds on estimator's mean square error applied to the passive near field source localization. More precisely, we focus on the so-called threshold prediction for which these bounds are known to be useful. We give closed form expressions of the McAulay-Seidman, the Hammersley-Chapman-Robbins, the McAulay-Hofstetter bounds and also, a recently proposed bound, the so-called Todros-Tabrikian bound, for the deterministic observation model (i.e., parameterized mean) and the stochastic observation model (i.e., parameterized covariance matrix). Finally, numerical simulations are given to assess the efficiency of these lower bounds to approximate the estimator's mean square error and to predict the threshold effect.
\end{abstract}

Index Terms - Near field source localization, performance analysis, mean square error, threshold prediction, deterministic lower bounds.

\section{INTRODUCTION}

Source localization is an important and challenging topic with several applications such as sonar, seismology, digital communications, etc. Particularly, the context of far field sources has been widely investigated in the literature and a plethora of algorithms to estimate localization parameters have been proposed [1]. In this case, the sources are assumed to be far from the array of sensors. Consequently, the propagating waves are assumed to have planar wavefront. However, when the sources are located in the so-called near field region, the curvature of the waves impinging on the sensors can no longer be approximated. Therefore, in this scenario, each source is characterized by its bearing and its range (distance between the source and a reference sensor). One can note the existence of some estimation algorithms adapted to the passive near field source localization [2]-[6].

Nevertheless, there exist only few works studying the asymptotic estimation performance in this context [4], [7] (by asymptotic we mean a large signal to noise ratio or a large number of snapshots [8], [9]). More precisely, to characterize the asymptotic performance of an estimator in terms of the mean square error, the Cramér-Rao 
bound, which is a tight bound under certain mild/general conditions [10], is the most popular tool [11]. However, the Cramér-Rao bound becomes too optimistic in the non-asymptotic region (i.e., when the outlier effect appears [12], [13].) This non-asymptotic region is delimited by the so-called threshold or breakdown point (i.e., when the estimator's mean square error increases dramatically.) One should note that the prediction of this threshold is of great importance since it delimits the optimal operating zone of the estimators.

To fill this lack, some other minimal bounds tighter than the Cramér-Rao bound has been proposed. In [14][17] the authors provide a different unification of some well known lower bounds on the mean square error of unbiased estimators of deterministic parameters. More precisely, in [16], Forster and Larzabal, solved the problem of establishing lower bounds on the mean square error for one deterministic parameter estimation using a constrained optimization problem. By imposing some adequate constraints on the bias for the considered optimization problem, they have rediscovered several lower bounds as the Cramér-Rao, the Barankin and the Bhattacharyya bounds. The extension to several unknown deterministic parameters can be found in [17]. In [14], Todros and Tabrikian propose a new class of performance lower bounds using the so-called integral transform which generalizes the derivative applied on the likelihood-ratio function. Thus, they showed that some well known lower bounds (as the Cramér-Rao, the McAulay-Seidman and the Bhattacharyya bounds) are obtained by a proper choice of the kernel of the integral transform of the likelihood-ratio function. It can be noted that the limiting expression (w.r.t. test points) of some of these lower bounds leads to the Barankin bound [18]. This bound is considered as the greatest lower bound on the mean square error of any unbiased estimator [17]. Unfortunately, the Barankin bound is the solution of an integral equation with a incomputable analytic solution.

To the best of our knowledge, no results can be found in the literature concerning the threshold prediction in the context of near field source localization ${ }^{1}$. In this correspondence, we fill this lack. We consider the two classical source signal model assumptions [9]: the deterministic model (i.e., when the signals are assumed to be deterministic) and the stochastic model (i.e., when the signals are assumed to be driven by a Gaussian random process). Furthermore, in both cases, the observation model is corrupted by a spatially colored noise. For each model, we propose to characterize the threshold region using some deterministic lower bounds on the estimator's mean square error (i.e., lower bounds w.r.t. unknown deterministic parameters of interest). In particular, we derive and analyze the following deterministic lower bounds: the McAulay-Seidman [20], the Hammersley-Chapman-Robbins [21], [22], the McAulay-Hofstetter [23] bounds and also, a new proposed bound, the so-called Todros-Tabrikian bound [14].

This correspondence is organized as follows. Section II formulates the problem and basic assumptions. In Section III we present the derivation of the lower bounds under the deterministic and stochastic assumption. Section

\footnotetext{
${ }^{1}$ Note that in [19] the authors analyzed only the stochastic signal model while both deterministic and stochastic signals models are analyzed in this paper. Moreover, the authors in [19] studied localization performance with $H$ distributed array of sensors. While, as mentioned by the authors, the source is near-field with respect to the overall "array of arrays". However, the far-field approximation was considered with respect to each array. Our model corresponds to $H=1$ and, in this case (only one array), the source cannot be considered in the far-field and in the near-field.
} 
IV is devoted to numerical analysis. Finally, conclusions are given in Section V

\section{MOdel SETUP}

In the near field context, the waves impinging on the sensors are considered to be spherical. Consequently, the time delay $\tau_{n}$ associated with the signal propagation time from a referential sensor (let say the first one) to the $(n+1)$ th sensor is given by (see Fig. 1 from [4] for the adequate labelling):

$$
\tau_{n}=\frac{2 \pi r}{\lambda}\left(\sqrt{1+\frac{n^{2} d^{2}}{r^{2}}-\frac{2 n d \sin \theta}{r}}-1\right),
$$

where $\lambda$ is the signal wavelength and where $r$ and $\theta \in[0, \pi / 2]$ denote the range (i.e., the distance between the source and the referential sensor) and the bearing of the source, respectively. More precisely, when the source is located in the so-called Fresnel region [3], i.e., if

$$
0.62\left(d^{3}(N-1)^{3} / \lambda\right)^{1 / 2}<r<2 d^{2}(N-1)^{2} / \lambda,
$$

in which $N$ denotes the number of sensors. Then, the time delay (1) is given by: $\tau_{n}=\omega n+\phi n^{2}+O\left(\frac{d^{2}}{r^{2}}\right)$, where $O\left(\frac{d^{2}}{r^{2}}\right)$ denotes the terms smaller or equal to $\frac{d^{2}}{r^{2}}$, in which the so-called electrical angles are given by:

$$
\omega=-2 \pi \frac{d}{\lambda} \sin (\theta)
$$

and

$$
\phi=\pi \frac{d^{2}}{\lambda r} \cos ^{2}(\theta)
$$

Neglecting $O\left(\frac{d^{2}}{r^{2}}\right)$, the time delay $\tau_{n}$ can be approximated by the following quadratic form

$$
\tau_{n}=\omega n+\phi n^{2}
$$

Consequently, considering a uniform and linear array (ULA) composed of $N$ sensors with an inter-element spacing $d$, receiving a single near-field and narrowband source, the observation model is, then, given as follows [2]:

$$
\mathbf{x}(t)=\left[x_{1}(t) \ldots x_{N}(t)\right]^{\ddagger}=\mathbf{a}(\omega, \phi) s(t)+\mathbf{v}(t), \quad t=1, \ldots, T,
$$

where $\ddagger$ denotes the transpose sign and $T$ is the number of snapshots, whereas, $\mathbf{v}(t)=\left[v_{1}(t) \ldots v_{N}(t)\right]^{\ddagger}$ and $x_{n}(t)$ is the observed signal at the output of the $(n+1)$ th sensor. The source signal is denoted by $s(t)$. The random process $v_{n}(t)$ is an additive noise. The $(n+1)$ th element of the steering vector $\mathbf{a}(\omega, \phi)$ is given by $[\mathbf{a}(\omega, \phi)]_{n+1}=e^{j\left(\omega n+\phi n^{2}\right)}$.

In the remain of this paper, we will use the following assumptions:

- The noise will be assumed to be a complex circular Gaussian process with zero mean with a known covariance (full rank) matrix $\boldsymbol{\Sigma}_{\text {noise }}$.

- For both deterministic [8] and stochastic [24] models, the unknown vector parameter is given by $\boldsymbol{\xi}=[\omega \phi]^{\ddagger}$. 
In the following $\boldsymbol{\xi}_{0}, \omega_{0}$ and $\phi_{0}$ represent the real value of the candidate parameters $\boldsymbol{\xi}, \omega$ and $\phi$, respectively. The joint probability density function of the observations $\chi=\left[\mathbf{x}^{\ddagger}(1) \ldots \mathbf{x}^{\ddagger}(T)\right]^{\ddagger} \sim \mathcal{C N}\left(\boldsymbol{\mu}\left(\boldsymbol{\xi}_{0}\right), \boldsymbol{\Sigma}\left(\boldsymbol{\xi}_{0}\right)\right)$ for a given $\boldsymbol{\xi}_{0}$, is expressed as:

$$
p\left(\boldsymbol{\chi} ; \boldsymbol{\xi}_{0}\right)=\frac{1}{\pi^{N T}\left|\boldsymbol{\Sigma}\left(\boldsymbol{\xi}_{0}\right)\right|} e^{-\left(\boldsymbol{\chi}-\boldsymbol{\mu}\left(\boldsymbol{\xi}_{0}\right)\right)^{H} \boldsymbol{\Sigma}\left(\boldsymbol{\xi}_{0}\right)^{-1}\left(\boldsymbol{\chi}-\boldsymbol{\mu}\left(\boldsymbol{\xi}_{0}\right)\right)},
$$

in which $|$.$| denotes the determinate operator. Depending on the considered signal model we will specify, in the$ following, the structure of $\boldsymbol{\mu}\left(\boldsymbol{\xi}_{0}\right)$ and $\boldsymbol{\Sigma}\left(\boldsymbol{\xi}_{0}\right)$.

\section{DETERMINISTIC LOWER BOUNDS DERIVATION}

\section{A. Background : Deterministic lower bounds unification}

The unification presented in [14] states that the mean square error (MSE) of any unbiased estimator can be lower bounded as follows:

$$
\operatorname{MSE}\left(\boldsymbol{\xi}_{0}\right)=E_{\boldsymbol{\chi} ; \boldsymbol{\xi}_{0}}\left\{\left(\hat{\boldsymbol{\xi}}-\boldsymbol{\xi}_{0}\right)\left(\hat{\boldsymbol{\xi}}-\boldsymbol{\xi}_{0}\right)^{\ddagger}\right\} \succcurlyeq \boldsymbol{C}=\boldsymbol{\Gamma} \boldsymbol{K}^{-1} \boldsymbol{\Gamma}^{H} \quad \text { with } \quad \boldsymbol{K}=E_{\boldsymbol{\chi} ; \boldsymbol{\xi}_{0}}\left\{\boldsymbol{\gamma} \boldsymbol{\gamma}^{H}\right\},
$$

where $\hat{\boldsymbol{\xi}}$ is the estimate of the true value parameter $\boldsymbol{\xi}_{0}, E_{\boldsymbol{\chi} ; \boldsymbol{\xi}_{0}}\{$.$\} is the expectation w.r.t. p\left(\boldsymbol{\chi} ; \boldsymbol{\xi}_{0}\right), \boldsymbol{C}$ is a lower bound matrix, $\boldsymbol{A} \succcurlyeq \boldsymbol{B}$ means that the matrix $\boldsymbol{A}-\boldsymbol{B}$ is non-negative defined. Consequently, it has been shown that for a specific choice of the couple $(\boldsymbol{\Gamma}, \gamma)$, one obtains a specific lower bound. In this way, the Cramér-Rao bound (CRB) can be defined using the following couple:

$$
\left\{\begin{array}{l}
\boldsymbol{\Gamma}_{\mathrm{CRB}}=\mathbf{1}_{2} \\
\gamma_{\mathrm{CRB}}=\left.\frac{\partial \operatorname{Ln} p(\boldsymbol{\chi} ; \boldsymbol{\xi})}{\partial \boldsymbol{\xi}}\right|_{\boldsymbol{\xi}=\boldsymbol{\xi}_{0}},
\end{array}\right.
$$

in which Ln denotes the natural logarithm where $\mathbf{1}_{l}$ is the $l \times 1$ vector filled by ones. For the following couple:

$$
\left\{\begin{array}{l}
\boldsymbol{\Gamma}_{\mathrm{MSB}}=\boldsymbol{\Phi}, \\
\gamma_{\mathrm{MSB}}=\left[\nu\left(\boldsymbol{\chi} ; \boldsymbol{\xi}_{1}\right) \ldots \nu\left(\boldsymbol{\chi} ; \boldsymbol{\xi}_{L}\right)\right]^{\ddagger},
\end{array}\right.
$$

one obtains the McAulay-Seidman bound (MSB), in which the so-called ratio-likelihood function is given by:

$$
\nu\left(\boldsymbol{x} ; \boldsymbol{\xi}_{l}\right)=\frac{p\left(\boldsymbol{\chi} ; \boldsymbol{\xi}_{l}\right)}{p\left(\boldsymbol{\chi} ; \boldsymbol{\xi}_{0}\right)}
$$

and where $\boldsymbol{\xi}_{l}$ for $l=1, \ldots, L$ denotes the $L$ test points, whereas $\boldsymbol{\Phi}=\left[\boldsymbol{\xi}_{1}-\boldsymbol{\xi}_{0} \ldots \boldsymbol{\xi}_{L}-\boldsymbol{\xi}_{0}\right]^{\ddagger}$. The HammersleyChapman-Robbins bound (HCRB) can be defined using

$$
\left\{\begin{array}{l}
\boldsymbol{\Gamma}_{\mathrm{HCRB}}=\left[\begin{array}{ll}
\mathbf{0}_{2} & \boldsymbol{\Phi}
\end{array}\right], \\
\boldsymbol{\gamma}_{\mathrm{HCRB}}=\left[\begin{array}{ll}
1 & \gamma_{\mathrm{MSB}}
\end{array}\right]^{\ddagger},
\end{array}\right.
$$

where $\mathbf{0}_{l}$ denotes the $l \times 1$ vector filled by zeros. Finally, one can define the McAulay-Hofstetter bound (MHB) using:

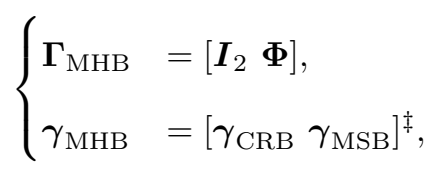


where $\boldsymbol{I}_{2}$ denotes the $2 \times 2$ identity matrix.

Recently, a new deterministic Cramér-Rao Fourier bound, called the Todros-Tabrikian Bound (TTB), was proposed in [14]. To have a gain in computing time, this latter applies the discrete Fourier transform (DFT) on $\mathbf{\Phi}$ and $\gamma_{\mathrm{MSB}}$. Consequently, it is given thanks to the following couple:

$$
\left\{\begin{array}{l}
\boldsymbol{\Gamma}_{\mathrm{TTB}}=\left[\boldsymbol{I}_{2} \boldsymbol{\Phi} \boldsymbol{W}^{H}\right], \\
\boldsymbol{\gamma}_{\mathrm{TTB}}=\left[\boldsymbol{\gamma}_{\mathrm{CRB}} \boldsymbol{\gamma}_{\mathrm{MSB}} \boldsymbol{W}^{\ddagger}\right]^{\ddagger},
\end{array}\right.
$$

where, in the near field context, the bi-dimensional discrete Fourier transform matrix $\boldsymbol{W}$ is given by

$$
[\boldsymbol{W}]_{p, l}=\exp \left(-i \boldsymbol{\Omega}_{p}^{\ddagger} \boldsymbol{\xi}_{l}\right)
$$

in which $\boldsymbol{\Omega}_{p}$ is expressed for the $p$ th frequency test bin $\boldsymbol{f}_{p}=\left[\begin{array}{ll}f_{p} & f_{p}^{\prime}\end{array}\right]^{\ddagger}$ as

$$
\boldsymbol{\Omega}_{p}=\left[\begin{array}{ll}
\frac{2 \pi f_{p}}{\Delta_{\omega} L_{1}} & \frac{2 \pi f_{p}^{\prime}}{\Delta_{\phi} L_{2}}
\end{array}\right]^{\ddagger}
$$

where $L=L_{1} L_{2}, f_{p} \in\left\{1, \ldots, L_{1}\right\}$ and $f_{p}^{\prime} \in\left\{1, \ldots, L_{2}\right\}$ in which $L_{1}$ and $L_{2}$ are the numbers of test points w.r.t. $\omega$ and $\phi$, respectively. $\Delta_{\omega}$ and $\Delta_{\phi}$ denote the uniform inter-test points w.r.t. $\omega$ and $\phi$, respectively. Consequently, the index $p$ is a unique combination of $\left\{f_{p}, f_{p}^{\prime}\right\}$ where the total number of these combinations is denoted by $P$.

One should note that the aforementioned bounds depend generally on the number of test points $L$ and/or the number of frequency test-bins $P$. Thus, in the following these bounds are indexed by $L$ and/or $P$. Next, we give matrix expressions of $C_{\mathrm{TTB}}^{(L, P)}$, then, $\boldsymbol{C}_{\mathrm{MSB}}^{(L)}, \boldsymbol{C}_{\mathrm{HCRB}}^{(L)}$ and $\boldsymbol{C}_{\mathrm{MHB}}^{(L)}$ will be deduced.

\section{B. Deterministic lower bounds matrix expressions}

After some straightforward calculation it can be shown (see [14, Appendix M]) that the TTB is expressed as

$$
\boldsymbol{C}_{\mathrm{TTB}}^{(L, P)}=\boldsymbol{C}_{\mathrm{CRB}}+\boldsymbol{Q} \boldsymbol{W}^{H}\left(\boldsymbol{W} \boldsymbol{R} \boldsymbol{W}^{H}\right)^{-1} \boldsymbol{W} \boldsymbol{Q}^{\ddagger}
$$

where

$$
\boldsymbol{Q}=\boldsymbol{C}_{\mathrm{CRB}} \boldsymbol{D}-\boldsymbol{\Phi}
$$

in which

$$
\boldsymbol{D}=\left[\begin{array}{lll}
d\left(\xi_{1}\right) & \ldots & d\left(\xi_{L}\right)
\end{array}\right]
$$

and

$$
\boldsymbol{d}\left(\boldsymbol{\xi}_{l}\right)=-\left.\left(\frac{\partial \operatorname{KLD}\left(p\left(\boldsymbol{\chi} ; \boldsymbol{\xi}_{l}\right) \| p(\boldsymbol{\chi} ; \boldsymbol{\xi})\right)}{\partial \boldsymbol{\xi}}\right)^{\ddagger}\right|_{\boldsymbol{\xi}=\boldsymbol{\xi}_{0}}
$$

where the KLD denotes the Kullback-Leibler divergence and

$$
\boldsymbol{R}=\boldsymbol{\Psi}-\boldsymbol{D}^{\ddagger} \boldsymbol{C}_{\mathrm{CRB}} \boldsymbol{D} \text { with }[\boldsymbol{\Psi}]_{m, n}=E_{\boldsymbol{\chi} ; \boldsymbol{\xi}_{0}}\left\{v\left(\boldsymbol{\chi}, \boldsymbol{\xi}_{m}\right) v\left(\boldsymbol{\chi}, \boldsymbol{\xi}_{n}\right)\right\} \text { where } m=1, \ldots, L, n=1, \ldots, L
$$


Following the same methodology, one can easily obtain the following matrix expressions:

$$
\begin{aligned}
& C_{\mathrm{MSB}}^{(L)}=\boldsymbol{\Phi} \boldsymbol{\Psi}^{-1} \boldsymbol{\Phi}^{\ddagger}, \\
& C_{\mathrm{HCRB}}^{(L)}=\boldsymbol{\Phi}\left(\boldsymbol{\Psi}-\mathbf{1}_{L} \mathbf{1}_{L}^{\ddagger}\right)^{-1} \boldsymbol{\Phi}^{\ddagger}, \\
& \boldsymbol{C}_{\mathrm{MHB}}^{(L)}=\boldsymbol{C}_{\mathrm{CRB}}+\boldsymbol{Q} \boldsymbol{R}^{-1} \boldsymbol{Q}^{\ddagger} .
\end{aligned}
$$

In the following we give closed-form expressions of the elements of $C_{\mathrm{MHB}}^{(L)}, C_{\mathrm{MHCB}}^{(L)}, C_{\mathrm{MSB}}^{(L)}$ and $C_{\mathrm{TTB}}^{(L, P)}$. We focus only on $D$ and $\Psi$, since the expression of $C_{\mathrm{CRB}}$ is well known, where for complex circular Gaussian observations (i.e., if $\chi \sim \mathcal{C N}\left(\boldsymbol{\mu}\left(\boldsymbol{\xi}_{0}\right), \boldsymbol{\Sigma}\left(\boldsymbol{\xi}_{0}\right)\right)$ ) one has $\boldsymbol{C}_{\mathrm{CRB}}=\mathbf{F I M}^{-1}$ [25], where

$$
[\mathbf{F I M}]_{i, k}=\operatorname{tr}\left\{\left.\left.\boldsymbol{\Sigma}\left(\boldsymbol{\xi}_{0}\right)^{-1} \frac{\partial \boldsymbol{\Sigma}(\boldsymbol{\xi})}{\partial[\boldsymbol{\xi}]_{i}}\right|_{\boldsymbol{\xi}=\boldsymbol{\xi}_{0}} \boldsymbol{\Sigma}\left(\boldsymbol{\xi}_{0}\right)^{-1} \frac{\partial \boldsymbol{\Sigma}(\boldsymbol{\xi})}{\partial[\boldsymbol{\xi}]_{k}}\right|_{\boldsymbol{\xi}=\boldsymbol{\xi}_{0}}\right\}+2 \Re\left\{\left.\left.\frac{\partial \boldsymbol{\mu}(\boldsymbol{\xi})^{H}}{\partial[\boldsymbol{\xi}]_{i}}\right|_{\boldsymbol{\xi}=\boldsymbol{\xi}_{0}} \boldsymbol{\Sigma}\left(\boldsymbol{\xi}_{0}\right)^{-1} \frac{\partial \boldsymbol{\mu}(\boldsymbol{\xi})}{\partial[\boldsymbol{\xi}]_{k}}\right|_{\boldsymbol{\xi}=\boldsymbol{\xi}_{0}}\right\}
$$

in which $\operatorname{tr}\{$.$\} and \Re\{$.$\} denote the trace operator and the real part, respectively.$

1) The deterministic case: In the deterministic case we assume that $s(t)=\alpha(t) e^{j\left(2 \pi f_{0} t+\psi(t)\right)}$ is the source signal with a carrier frequency equal to $f_{0}$ where $\alpha(t)$ and $\psi(t)$ are the known real amplitude and the known shift phase, respectively. Consequently, one has an observation model with a parameterized mean such that $\chi \sim$ $\mathcal{C N}\left(\boldsymbol{\mu}\left(\boldsymbol{\xi}_{0}\right), \boldsymbol{\Sigma}_{\text {noise }}\right)$ where $\boldsymbol{\mu}\left(\boldsymbol{\xi}_{0}\right)=\left[s(1) \mathbf{a}^{\ddagger}\left(\omega_{0}, \phi_{0}\right) \ldots s(T) \mathbf{a}^{\ddagger}\left(\omega_{0}, \phi_{0}\right)\right]^{\ddagger}$. Then, by applying (25) one has

$$
[\mathbf{F I M}]_{i, k}=2 \Re\left\{\left.\left.\frac{\partial \boldsymbol{\mu}(\boldsymbol{\xi})^{H}}{\partial[\boldsymbol{\xi}]_{i}}\right|_{\boldsymbol{\xi}=\boldsymbol{\xi}_{0}} \boldsymbol{\Sigma}_{\text {noise }}^{-1} \frac{\partial \boldsymbol{\mu}(\boldsymbol{\xi})}{\partial[\boldsymbol{\xi}]_{k}}\right|_{\boldsymbol{\xi}=\boldsymbol{\xi}_{0}}\right\}, i=1,2, \quad k=1,2 .
$$

Furthermore, one obtains:

$$
\begin{aligned}
\operatorname{KLD}\left(p\left(\boldsymbol{\chi} ; \boldsymbol{\xi}_{n}\right) \| p(\boldsymbol{\chi} ; \boldsymbol{\xi})\right) & =\int_{\mathbb{C}^{N T}} p\left(\boldsymbol{\chi} ; \boldsymbol{\xi}_{n}\right) \operatorname{Ln} \frac{p\left(\boldsymbol{\chi} ; \boldsymbol{\xi}_{n}\right)}{p(\boldsymbol{\chi} ; \boldsymbol{\xi})} d \boldsymbol{\chi} \\
& =\int_{\mathbb{C}^{N T}}\left[(\boldsymbol{\chi}-\boldsymbol{\mu}(\boldsymbol{\xi}))^{H} \boldsymbol{\Sigma}_{\text {noise }}^{-1}(\boldsymbol{\chi}-\boldsymbol{\mu}(\boldsymbol{\xi}))-\left(\boldsymbol{\chi}-\boldsymbol{\mu}\left(\boldsymbol{\xi}_{n}\right)\right)^{H} \boldsymbol{\Sigma}_{\text {noise }}^{-1}\left(\boldsymbol{\chi}-\boldsymbol{\mu}\left(\boldsymbol{\xi}_{n}\right)\right)\right] p\left(\boldsymbol{\chi} ; \boldsymbol{\xi}_{n}\right) d \boldsymbol{\chi} \\
& =\int_{\mathbb{C}^{N T}}\left[\chi^{H} \boldsymbol{\Sigma}_{\text {noise }}^{-1}\left(\boldsymbol{\mu}\left(\boldsymbol{\xi}_{n}\right)-\boldsymbol{\mu}(\boldsymbol{\xi})\right)-\boldsymbol{\mu}(\boldsymbol{\xi})^{H} \boldsymbol{\Sigma}_{\text {noise }}^{-1}(\boldsymbol{\chi}-\boldsymbol{\mu}(\boldsymbol{\xi}))\right] f\left(\boldsymbol{\chi} ; \boldsymbol{\xi}_{n}\right) d \boldsymbol{\chi} \\
& =\left(\boldsymbol{\mu}\left(\boldsymbol{\xi}_{n}\right)-\boldsymbol{\mu}(\boldsymbol{\xi})\right)^{H} \boldsymbol{\Sigma}_{\text {noise }}^{-1}\left(\boldsymbol{\mu}\left(\boldsymbol{\xi}_{n}\right)-\boldsymbol{\mu}(\boldsymbol{\xi})\right) .
\end{aligned}
$$

The term $\operatorname{KLD}\left(p\left(\boldsymbol{\chi} ; \boldsymbol{\xi}_{n}\right) \| p(\boldsymbol{\chi} ; \boldsymbol{\xi})\right)$ and (20)-(21) lead to :

$$
\begin{aligned}
& {[\Psi]_{m, n}=\int_{\mathbb{C}^{N T}} \frac{1}{\pi\left|\boldsymbol{\Sigma}_{\text {noise }}\right|} \times} \\
& \exp \left(-\left(\boldsymbol{\chi}-\boldsymbol{\mu}\left(\boldsymbol{\xi}_{m}\right)\right)^{H} \boldsymbol{\Sigma}_{\text {noise }}^{-1}\left(\boldsymbol{\chi}-\boldsymbol{\mu}\left(\boldsymbol{\xi}_{m}\right)\right)-\left(\boldsymbol{\chi}-\boldsymbol{\mu}\left(\boldsymbol{\xi}_{n}\right)\right)^{H} \boldsymbol{\Sigma}_{\text {noise }}^{-1}\left(\boldsymbol{\chi}-\boldsymbol{\mu}\left(\boldsymbol{\xi}_{n}\right)\right)+2\left(\boldsymbol{\chi}-\boldsymbol{\mu}\left(\boldsymbol{\xi}_{0}\right)\right)^{H} \boldsymbol{\Sigma}_{\text {noise }}^{-1}\left(\boldsymbol{\chi}-\boldsymbol{\mu}\left(\boldsymbol{\xi}_{0}\right)\right)\right) d \boldsymbol{\chi} \\
& =\frac{\alpha\left(\boldsymbol{\xi}_{m}, \boldsymbol{\xi}_{n}\right)}{\pi\left|\boldsymbol{\Sigma}_{\text {noise }}\right|} \underbrace{\int_{\mathbb{C}^{N T}} \exp -\left(\boldsymbol{\chi}-\boldsymbol{\mu}\left(\boldsymbol{\xi}_{m}\right)-\boldsymbol{\mu}\left(\boldsymbol{\xi}_{n}\right)+\boldsymbol{\mu}\left(\boldsymbol{\xi}_{0}\right)\right)^{H} \boldsymbol{\Sigma}_{\text {noise }}^{-1}\left(\boldsymbol{\chi}-\boldsymbol{\mu}\left(\boldsymbol{\xi}_{m}\right)-\boldsymbol{\mu}\left(\boldsymbol{\xi}_{n}\right)+\boldsymbol{\mu}\left(\boldsymbol{\xi}_{0}\right)\right) d \boldsymbol{\chi}}_{\pi \mid \boldsymbol{\Sigma}_{\text {noise }}}=\alpha\left(\boldsymbol{\xi}_{m}, \boldsymbol{\xi}_{n}\right),
\end{aligned}
$$

in which

$$
\begin{aligned}
& \alpha\left(\boldsymbol{\xi}_{m}, \boldsymbol{\xi}_{n}\right)=\exp \left(-2 \boldsymbol{\mu}\left(\boldsymbol{\xi}_{0}\right)^{H} \boldsymbol{\Sigma}_{\text {noise }}^{-1} \boldsymbol{\mu}\left(\boldsymbol{\xi}_{0}\right)-\boldsymbol{\mu}\left(\boldsymbol{\xi}_{0}\right)^{H} \boldsymbol{\Sigma}_{\text {noise }}^{-1}\left(\boldsymbol{\mu}\left(\boldsymbol{\xi}_{m}\right)+\boldsymbol{\mu}\left(\boldsymbol{\xi}_{n}\right)\right)\right. \\
& \left.+\boldsymbol{\mu}\left(\boldsymbol{\xi}_{m}\right)^{H} \boldsymbol{\Sigma}_{\text {noise }}^{-1}\left(\boldsymbol{\mu}\left(\boldsymbol{\xi}_{n}\right)-\boldsymbol{\mu}\left(\boldsymbol{\xi}_{0}\right)\right)+\boldsymbol{\mu}\left(\boldsymbol{\xi}_{n}\right)^{H} \boldsymbol{\Sigma}_{\text {noise }}^{-1}\left(\boldsymbol{\mu}\left(\boldsymbol{\xi}_{m}\right)-\boldsymbol{\mu}\left(\boldsymbol{\xi}_{0}\right)\right)\right)=\exp \left(\left(\boldsymbol{\mu}\left(\boldsymbol{\xi}_{n}\right)-\boldsymbol{\mu}\left(\boldsymbol{\xi}_{0}\right)\right)^{H} \boldsymbol{\Sigma}_{\text {noise }}^{-1}\left(\boldsymbol{\mu}\left(\boldsymbol{\xi}_{m}\right)-\boldsymbol{\mu}\left(\boldsymbol{\xi}_{0}\right)\right)\right) .
\end{aligned}
$$


Finally, $C_{\mathrm{MHB}}^{(L)}, C_{\mathrm{MHCB}}^{(L)}, C_{\mathrm{MSB}}^{(L)}$ and $C_{\mathrm{TTB}}^{(L, P)}$ are given by plugging (26), (27) and (28) into (24), (23), (22) and (17), respectively. See Appendix A for non-matrix expressions of $C_{\mathrm{TTB}}^{(L, 1)}$ (i.e., in the case where $P=1$.)

2) The stochastic case: Let us consider the stochastic model, i.e., when the signals are assumed to be Gaussian (with zero mean and variance $\sigma_{s}^{2}$ ) independent of the noise. Under this assumption, one obtains an observation model with a parameterized covariance matrix such that $\chi \sim \mathcal{C N}\left(\mathbf{0}, \boldsymbol{\Sigma}\left(\boldsymbol{\xi}_{0}\right)\right)$ where the covariance matrix $\boldsymbol{\Sigma}\left(\boldsymbol{\xi}_{0}\right)=$ $\sigma_{s}^{2} \boldsymbol{I}_{T} \otimes \mathbf{a}\left(\omega_{0}, \phi_{0}\right) \mathbf{a}^{H}\left(\omega_{0}, \phi_{0}\right)+\boldsymbol{\Sigma}_{\text {noise }}$ in which $\otimes$ denotes the Kronecker product. Consequently, the FIM in (25) becomes:

$$
[\mathbf{F I M}]_{i, k}=T \operatorname{tr}\left\{\left.\left.\boldsymbol{\Sigma}\left(\boldsymbol{\xi}_{0}\right)^{-1} \frac{\partial \boldsymbol{\Sigma}(\boldsymbol{\xi})}{\partial[\boldsymbol{\xi}]_{i}}\right|_{\boldsymbol{\xi}=\boldsymbol{\xi}_{0}} \boldsymbol{\Sigma}\left(\boldsymbol{\xi}_{0}\right)^{-1} \frac{\partial \boldsymbol{\Sigma}(\boldsymbol{\xi})}{\partial[\boldsymbol{\xi}]_{k}}\right|_{\boldsymbol{\xi}=\boldsymbol{\xi}_{0}}\right\}, i=1,2, k=1,2
$$

First, note that:

$$
\begin{aligned}
\operatorname{KLD}\left(p\left(\boldsymbol{\chi} ; \boldsymbol{\xi}_{n}\right) \| p(\boldsymbol{\chi} ; \boldsymbol{\xi})\right) & =\int_{\mathbb{C}^{N T}} p\left(\boldsymbol{\chi} ; \boldsymbol{\xi}_{n}\right) \operatorname{Ln} \frac{p\left(\boldsymbol{\chi} ; \boldsymbol{\xi}_{n}\right)}{p(\boldsymbol{\chi} ; \boldsymbol{\xi})} d \boldsymbol{\chi} \\
& =\int_{\mathbb{C}^{N T}} \frac{1}{\pi^{N T}\left|\boldsymbol{\Sigma}\left(\boldsymbol{\xi}_{n}\right)\right|}\left(\chi^{H}\left(\boldsymbol{\Sigma}\left(\boldsymbol{\xi}_{n}\right)^{-1}-\boldsymbol{\Sigma}(\boldsymbol{\xi})^{-1}\right) \chi\right) \exp \left(-\chi^{H} \boldsymbol{\Sigma}\left(\boldsymbol{\xi}_{n}\right)^{-1} \boldsymbol{\chi}\right) d \boldsymbol{\chi}+\operatorname{Ln} \frac{|\boldsymbol{\Sigma}(\boldsymbol{\xi})|}{\left|\boldsymbol{\Sigma}\left(\boldsymbol{\xi}_{n}\right)\right|} \\
& =E_{\boldsymbol{\chi} ; \boldsymbol{\xi}_{n}}\left\{\chi^{H} \boldsymbol{\Sigma}(\boldsymbol{\xi}) \boldsymbol{\chi}\right\}+E_{\boldsymbol{\chi} ; \boldsymbol{\xi}_{n}}\left\{\boldsymbol{\chi}^{H} \boldsymbol{\Sigma}\left(\boldsymbol{\xi}_{n}\right) \boldsymbol{\chi}\right\}+\operatorname{Ln} \frac{|\boldsymbol{\Sigma}(\boldsymbol{\xi})|}{\left|\boldsymbol{\Sigma}\left(\boldsymbol{\xi}_{n}\right)\right|}
\end{aligned}
$$

Observing that:

$$
E_{\boldsymbol{\chi} ; \boldsymbol{\xi}_{n}}\left\{\chi^{H} \boldsymbol{\Sigma}(\boldsymbol{\xi}) \chi\right\}=\sum_{i=1}^{N T} \sum_{j=1}^{N T} E_{\boldsymbol{\chi} ; \boldsymbol{\xi}_{n}}\left\{[\boldsymbol{\chi}]_{i}^{*}[\chi]_{j}\left[\boldsymbol{\Sigma}(\boldsymbol{\xi})^{-1}\right]_{i, j}\right\}=\sum_{j=1}^{N T}\left[\boldsymbol{\Sigma}\left(\boldsymbol{\xi}_{n}\right) \boldsymbol{\Sigma}(\boldsymbol{\xi})^{-1}\right]_{j, j}=\operatorname{tr}\left(\boldsymbol{\Sigma}\left(\boldsymbol{\xi}_{n}\right) \boldsymbol{\Sigma}(\boldsymbol{\xi})^{-1}\right)
$$

and, in the same way,

$$
E_{\chi ; \boldsymbol{\xi}_{n}}\left\{\chi^{H} \boldsymbol{\Sigma}\left(\boldsymbol{\xi}_{0}\right)^{-1} \chi\right\}=N T
$$

Thus, plugging (31) and (32) into (30) one obtains

$$
\operatorname{KLD}\left(p\left(\chi ; \boldsymbol{\xi}_{n}\right)|| f(\chi ; \boldsymbol{\xi})\right)=\operatorname{Ln} \frac{|\boldsymbol{\Sigma}|}{\left|\boldsymbol{\Sigma}\left(\boldsymbol{\xi}_{n}\right)\right|}+\operatorname{tr}\left(\boldsymbol{\Sigma}\left(\boldsymbol{\xi}_{n}\right) \boldsymbol{\Sigma}(\boldsymbol{\xi})^{-1}\right)+N T .
$$

Secondly, one has:

$$
\begin{aligned}
{[\boldsymbol{\Psi}]_{n, m} } & =\frac{\left|\boldsymbol{\Sigma}\left(\boldsymbol{\xi}_{0}\right)\right|}{\pi^{N T}\left|\boldsymbol{\Sigma}\left(\boldsymbol{\xi}_{m}\right)\right|\left|\boldsymbol{\Sigma}\left(\boldsymbol{\xi}_{n}\right)\right|} \underbrace{\int_{\mathbb{C}^{N T}} \exp \left(-\chi^{H}\left(\boldsymbol{\Sigma}\left(\boldsymbol{\xi}_{m}\right)^{-1}+\boldsymbol{\Sigma}\left(\boldsymbol{\xi}_{n}\right)^{-1}-\boldsymbol{\Sigma}\left(\boldsymbol{\xi}_{0}\right)^{-1}\right) \chi\right) d \chi}_{\pi^{N T}\left|\left(\boldsymbol{\Sigma}\left(\boldsymbol{\xi}_{m}\right)^{-1}+\boldsymbol{\Sigma}\left(\boldsymbol{\xi}_{n}\right)^{-1}-\boldsymbol{\Sigma}\left(\boldsymbol{\xi}_{0}\right)^{-1}\right)^{-1}\right|} \\
& =\frac{\left|\boldsymbol{\Sigma}\left(\boldsymbol{\xi}_{0}\right)\right|}{\left|\boldsymbol{\Sigma}\left(\boldsymbol{\xi}_{m}\right)\right|\left|\boldsymbol{\Sigma}\left(\boldsymbol{\xi}_{n}\right)\right|\left|\boldsymbol{\Sigma}\left(\boldsymbol{\xi}_{m}\right)^{-1}+\boldsymbol{\Sigma}\left(\boldsymbol{\xi}_{n}\right)^{-1}-\boldsymbol{\Sigma}\left(\boldsymbol{\xi}_{0}\right)^{-1}\right|}
\end{aligned}
$$

Consequently, using the fact that $\frac{\partial \operatorname{Ln}|\boldsymbol{\Sigma}(\boldsymbol{\xi})|}{\partial \boldsymbol{\xi}}=\operatorname{tr}\left\{\boldsymbol{\Sigma}(\boldsymbol{\xi})^{-1} \frac{\partial \boldsymbol{\Sigma}(\boldsymbol{\xi})}{\partial \boldsymbol{\xi}}\right\}$ [26] and plugging (29), (33) and (34) into (24), (23), (22) and (17) one obtains $C_{\mathrm{MHB}}^{(L)}, C_{\mathrm{MHCB}}^{(L)}, C_{\mathrm{MSB}}^{(L)}$ and $C_{\mathrm{TTB}}^{(L, P)}$, respectively. See Appendix A for non-matrix expressions of $C_{\mathrm{TTB}}^{(L, 1)}$ (i.e., in the case $P=1$.)

\section{NUMERICAL SIMULATIONS}

The scenario used in these simulations is a ULA of $N=5$ sensors spaced by $d=\frac{\lambda}{2}$. The noise is assumed to be a complex circular white Gaussian random process with zero-mean and known variance $\sigma^{2}$, uncorrelated both temporally and spatially. 


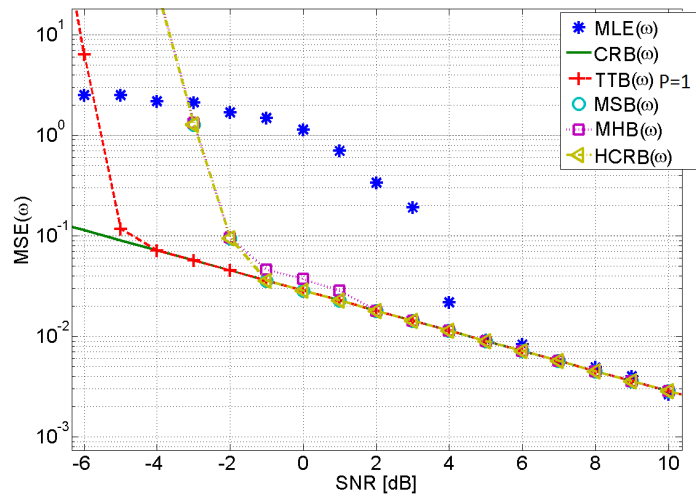

Fig. 1. Lower bounds on the mean square error (deterministic case) w.r.t. $\omega$ for near field source localization, with $T=10$ and $(\theta, r)=$ $\left(45^{\circ}, 6 \lambda\right)$.

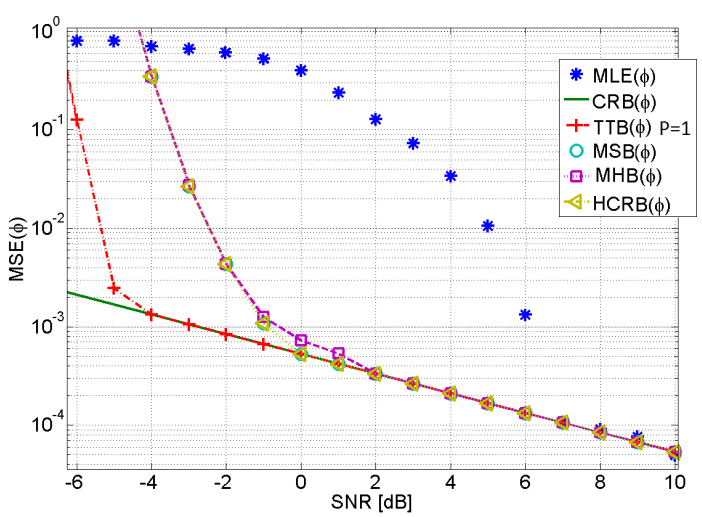

Fig. 2. Lower bounds on the mean square error (deterministic case) w.r.t. $\phi$ for near field source localization, with $T=10$ and $(\theta, r)=$ $\left(45^{\circ}, 6 \lambda\right)$.

To compare the threshold prediction accuracy we plot the MSE w.r.t. $\omega$ and $\phi$ using 1000 Monte Carlo trials. In both deterministic and stochastic cases (see Fig. 1-4), we compute $C_{\mathrm{MHB}}^{(L)}, C_{\mathrm{MHCB}}^{(L)}, C_{\mathrm{MSB}}^{(L)}$ using $L=2^{10}$ test points (more precisely, we used $L_{1}=2^{5}$ test points over the parameter $\omega$ and $L_{2}=2^{5}$ test points over the parameter $\phi$.) The TTB, $C_{\mathrm{TTB}}^{(L, 1)}$, is obtained using $L=2^{10}$ test points and also by numerical maximization over $2^{10}$ frequency test bins for $P=1$.

Threshold prediction: Fig. 1-4 provide an illustration of the usefulness of the aforementioned deterministic lower bounds in the case of deterministic and stochastic model assumptions for $\omega$ and $\phi$. First, one can notice that the MSE of $\phi$ is lower than the MSE of $\omega$ which is expected due the range of those parameters and from the fact that $\phi$ is the coefficient of the second order, whereas, $\omega$ is the coefficient of the first order w.r.t. the time delay (see (5)). Second, one can notice that all the aforementioned bounds provide a good prediction of the MSE threshold.

In Fig. 1-4, we considered only one frequency test bin. In this case we notice that the MHB is more accurate than the TTB. This degradation is due to the fact that the TTB is based on lossy compression of the samples of the likelihood ratio function into $P=1$ frequency test-bin (i.e., it considers only one constraints for $P=1$, see Appendix B), whereas the MHB does not apply this lossy compression and use all the information contained in the $L$ samples in the parameter space (i.e., $L$ constraints). Nevertheless, one can note that the advantage of the TTB is its computational cost (the computational complexity of the TTB is lower in comparison to the MSB, HCRB and MHB due to the inversion matrix, see (24), (23), (22) and (17).) Consequently, we can consider the maximization of the TTB over more than one frequency test bins. As shown in the following, this leads to an considerable improvement of the TTB.

Effect of the number of frequency test-bins on the TTB: Considering the maximization over more than only one frequency test bins is essential to ascertain the proper use of the TTB. For $P>1$ one can maximize the TTB via different numerical optimization methods. One of the commonly used approach is to consider a few fast 
one-dimensional search procedures. More precisely we use a numerical maximization approach under which the frequency test-bins $\boldsymbol{\Omega}_{1}, \ldots, \boldsymbol{\Omega}_{P}$ are selected in a sequential manner. In the first step, maximization is performed w.r.t. one test-bin $\boldsymbol{\Omega}_{1}$. In the $k$-th step $(k \leq P)$ maximization is performed w.r.t. $\boldsymbol{\Omega}_{k}$ while $\boldsymbol{\Omega}_{1}, \ldots, \boldsymbol{\Omega}_{k-1}$ are fixed. This sequential procedure is being continued until $k=P$.

As shown in Fig. 5, one can notice that this maximization, with respect to the frequency test-bins, leads to a considerable improvement of the threshold prediction (the threshold prediction is now only $2 \mathrm{~dB}$ far from the true value, instead of 8-10 dB without maximization). On the other hand, the TTB out performs the MHB (and consequently also the MSB and the HCRB) with a maximization of only $P=5$ frequency test-bins, or more, as illustrated in Fig. 5.

Effect of the number of sensors on the threshold: Fig. 6 shows us that the number of sensors has an important effect on the asymptotic variance of the MLE but also on the presence of outliers (which is deduced by the SNR value of the breakdown point). In this example, a decreasing of 5 sensors increases the SNR value of the breakdown point by approximatively $2 \mathrm{~dB}$ (i.e., outliers will appear $2 \mathrm{~dB}$ earlier if we remove 5 sensors)

Effect of the number of snapshots on the threshold: Finally, one should note that increasing the number of snapshots has a similar effect to decrease the noise variance or to increase the SNR as shown in Fig. 7. This can be also explained for the particular case of fixed and constant amplitude, i.e, $\boldsymbol{x}(t) \sim \mathcal{C N}(\boldsymbol{a}, \boldsymbol{\Sigma}),, t=1 \ldots T$. In this case, a sufficient statistic is to sum all the observations w.r.t. the snapshots. Since the amplitude is constant, thus, one obtains the following sufficient statistic $\chi=\frac{1}{T} \sum_{t=1}^{T} \boldsymbol{x}(t) \sim \mathcal{C N}\left(\boldsymbol{a}, \frac{\boldsymbol{\Sigma}}{T}\right)$, which is equivalent to reduce the variance by a coefficient equal to the number of snapshots.

\section{Conclusion}

In this paper, we present the derivation of different deterministic lower bounds on the MSE in a near field source localization context. This analysis allowed us to characterize the non-asymptotic performance estimators mean square error. In particular, we focused on the threshold/breakdown prediction. Furthermore, this study shows that the recently proposed TTB out performs its predecessors as the MHB by using only a few one-dimensional sequential maximization over frequency test-bins.

\section{APPENDIX A}

In this appendix, we give non-matrix expression of the TTB for the case $P=1$. In this case, the matrix $\boldsymbol{W}$ will be reduced to a row vector of dimension $L$, such that $[\boldsymbol{W}]_{1, l}=\exp \left(-j 2 \pi \frac{l-1}{L}\right), \quad l=1, \ldots, L$. Let $\rho=\boldsymbol{W} \boldsymbol{R} \boldsymbol{W}^{H}$, consequently, one has: $\rho=\sum_{m=1}^{L} \sum_{n=1}^{L}[\boldsymbol{R}]_{m, n} \exp \left(2 j \pi \frac{n-m}{L}\right)$. On the other hand, using (21), one obtains:

$$
[\boldsymbol{R}]_{m, n}=[\boldsymbol{\Psi}]_{m, n}-\left.\left.\sum_{p=1}^{2} \sum_{q=1}^{2}\left(\frac{\partial \operatorname{KLD}\left(p\left(\boldsymbol{x} ; \boldsymbol{\xi}_{m}\right)|| p(\boldsymbol{x} ; \boldsymbol{\xi})\right)}{\partial[\boldsymbol{\xi}]_{p}}\right)\right|_{\boldsymbol{\xi}=\boldsymbol{\xi}_{0}}\left[\boldsymbol{C}_{\mathrm{CRB}}\right]_{p, q}\left(\frac{\partial \operatorname{KLD}\left(p\left(\boldsymbol{x} ; \boldsymbol{\xi}_{n}\right)|| p(\boldsymbol{x} ; \boldsymbol{\xi})\right)}{\partial[\boldsymbol{\xi}]_{q}}\right)\right|_{\boldsymbol{\xi}=\boldsymbol{\xi}_{0}} .
$$

Consequently, one obtains the closed-form expression 


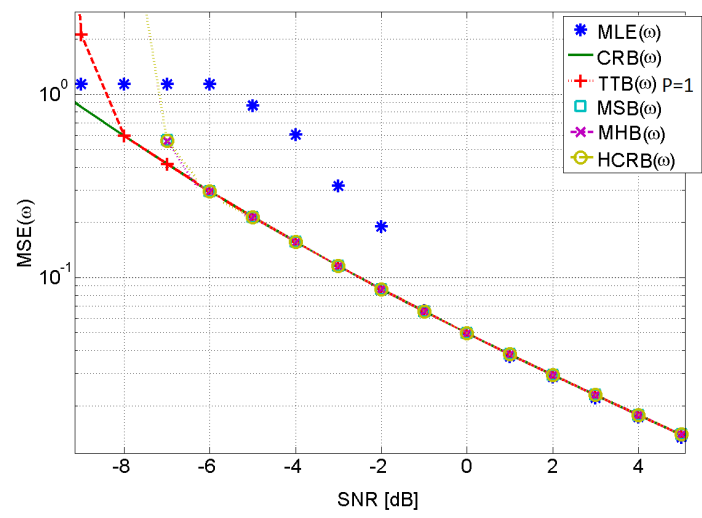

Fig. 3. Lower bounds on the mean square error (the stochastic case) w.r.t. $\omega$ for near field source localization, with $T=100$ and $(\theta, r)=$ $\left(30^{\circ}, 6 \lambda\right)$.

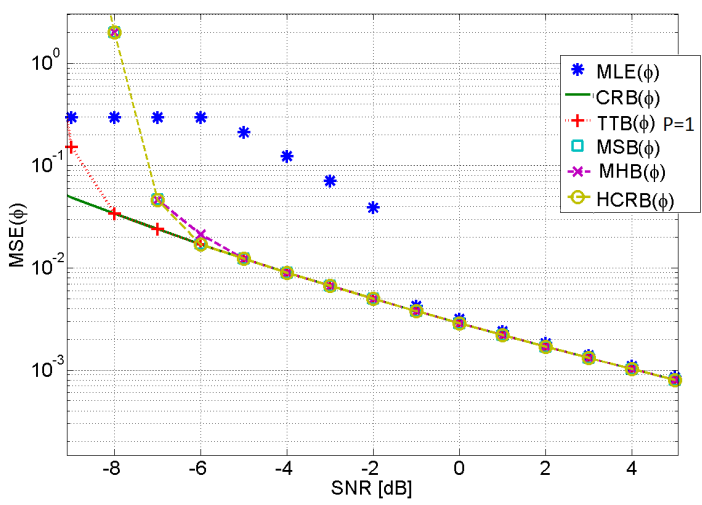

Fig. 4. Lower bounds on the mean square error (the stochastic case) w.r.t. $\phi$ for near field source localization, with $T=100$ and $(\theta, r)=$ $\left(30^{\circ}, 6 \lambda\right)$.

$$
\begin{gathered}
{\left[\boldsymbol{C}_{\mathrm{TTB}}^{(L, 1)}\right]_{p, q}=\left[\boldsymbol{C}_{\mathrm{CRB}}\right]_{p, q}+\frac{1}{\rho} \sum_{m=1}^{L} \sum_{n=1}^{L} \beta(p, m) \beta(q, n) \exp \left(j 2 \pi \frac{n-m}{L}\right),} \\
\text { in which } \beta(p, n)=\left.\sum_{q=1}^{2}\left[\boldsymbol{C}_{\mathrm{CRB}}\right]_{p, q}\left(\frac{\partial \operatorname{KLD}\left(p\left(\boldsymbol{x} ; \boldsymbol{\xi}_{n}\right) \| p(\boldsymbol{x} ; \boldsymbol{\xi})\right)}{\partial[\boldsymbol{\xi}]_{q}}\right)\right|_{\boldsymbol{\xi}=\boldsymbol{\xi}_{0}}+\left[\boldsymbol{\xi}_{0}\right]_{p}-\left[\boldsymbol{\xi}_{n}\right]_{p} .
\end{gathered}
$$

\section{A. The deterministic case:}

Plugging (27) and (28) into (36), one obtains

$$
\begin{aligned}
& {\left[\boldsymbol{C}_{\mathrm{TTB}}^{(L, 1)}\right]_{p, q}=\left[\boldsymbol{C}_{\mathrm{CRB}}\right]_{p, q}+\frac{\sum_{m=1}^{L} \sum_{n=1}^{L} \beta_{\mathrm{cond}}(p, m) \beta_{\mathrm{cond}}(q, n) \exp \left(j 2 \pi \frac{n-m}{L}\right)}{\sum_{m=1}^{L} \sum_{n=1}^{L} \gamma_{\text {cond }}(m, n) \exp \left(2 j \pi \frac{n-m}{L}\right)},} \\
& \text { where } \quad \gamma_{\text {cond }}(m, n)=\alpha\left(\boldsymbol{\xi}_{m}, \boldsymbol{\xi}_{n}\right)-\sum_{p=1}^{2} \sum_{q=1}^{2} \kappa_{\text {cond }}(m, p) \kappa_{\text {cond }}(n, q)\left[\boldsymbol{C}_{\mathrm{CRB}}\right]_{p, q} \text {, } \\
& \text { in which } \quad \kappa_{\text {cond }}(n, q)=\left.\left(\frac{\partial\left(\boldsymbol{\mu}\left(\boldsymbol{\xi}_{n}\right)-\boldsymbol{\mu}(\boldsymbol{\xi})\right)^{H} \boldsymbol{\Sigma}_{\text {noise }}^{-1}\left(\boldsymbol{\mu}\left(\boldsymbol{\xi}_{n}\right)-\boldsymbol{\mu}(\boldsymbol{\xi})\right)}{\partial[\boldsymbol{\xi}]_{q}}\right)\right|_{\boldsymbol{\xi}=\boldsymbol{\xi}_{0}} \\
& =-\dot{\boldsymbol{\mu}}_{q} \boldsymbol{\xi}_{0}^{H} \boldsymbol{\Sigma}_{\text {noise }}^{-1}\left(\boldsymbol{\mu}\left(\boldsymbol{\xi}_{n}\right)-\boldsymbol{\mu}(\boldsymbol{\xi})\right)+\left(\boldsymbol{\mu}\left(\boldsymbol{\xi}_{n}\right)-\boldsymbol{\mu}(\boldsymbol{\xi})\right)^{H} \boldsymbol{\Sigma}_{\text {noise }}^{-1}-\dot{\boldsymbol{\mu}}_{q}\left(\boldsymbol{\xi}_{0}\right)
\end{aligned}
$$

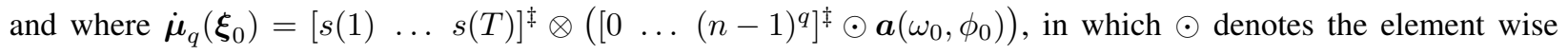
product and where $\beta_{\text {cond }}(p, n)=\left.\sum_{q=1}^{2}\left[\boldsymbol{C}_{\mathrm{CRB}}\right]_{p, q}\left(\frac{\partial \kappa_{\mathrm{cond}}(n, q)}{\partial[\boldsymbol{\xi}]_{q}}\right)\right|_{\boldsymbol{\xi}=\boldsymbol{\xi}_{0}}+\left[\boldsymbol{\xi}_{0}\right]_{p}-\left[\boldsymbol{\xi}_{n}\right]_{p}$.

\section{B. The stochastic case:}

Plugging (33) and (34) into (36), one obtains

$$
\left[\boldsymbol{C}_{\mathrm{TTB}}^{(L, 1)}\right]_{p, q}=\left[\boldsymbol{C}_{\mathrm{CRB}}\right]_{p, q}+\frac{\sum_{m=1}^{L} \sum_{n=1}^{L} \beta_{\text {uncond }}(p, m) \beta_{\text {uncond }}(q, n) \exp \left(j 2 \pi \frac{n-m}{L}\right)}{\sum_{m=1}^{L} \sum_{n=1}^{L} \gamma_{\text {uncond }}(m, n) \exp \left(2 j \pi \frac{n-m}{L}\right)},
$$




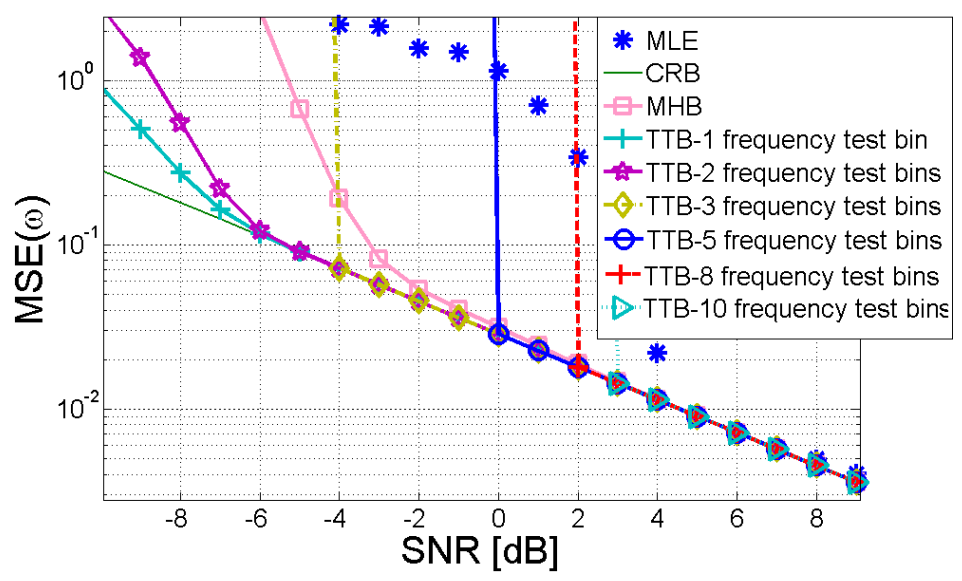

Fig. 5. Threshold prediction w.r.t. $\omega$ using a sequential maximization of the TTB with respect to the frequency test-bins with $L=2^{8}$.

where

$\gamma_{\text {uncond }}(m, n)=\frac{\left|\boldsymbol{\Sigma}\left(\boldsymbol{\xi}_{0}\right)\right|}{\left|\boldsymbol{\Sigma}\left(\boldsymbol{\xi}_{m}\right)\right|\left|\boldsymbol{\Sigma}\left(\boldsymbol{\xi}_{n}\right)\right|\left|\boldsymbol{\Sigma}\left(\boldsymbol{\xi}_{m}\right)^{-1}+\boldsymbol{\Sigma}\left(\boldsymbol{\xi}_{n}\right)^{-1}-\boldsymbol{\Sigma}\left(\boldsymbol{\xi}_{0}\right)^{-1}\right|}-\sum_{p=1}^{2} \sum_{q=1}^{2} \kappa_{\text {uncond }}(m, p) \kappa_{\text {uncond }}(n, q)\left[\boldsymbol{C}_{\mathrm{CRB}}\right]_{p, q}$.

in which

$$
\begin{aligned}
\kappa_{\text {uncond }}(n, q)= & \left.\left(\frac{\partial\left(\operatorname{Ln} \frac{|\boldsymbol{\Sigma}(\boldsymbol{\xi})|}{\left|\boldsymbol{\Sigma}\left(\boldsymbol{\xi}_{n}\right)\right|}+\operatorname{tr}\left(\boldsymbol{\Sigma}\left(\boldsymbol{\xi}_{n}\right) \boldsymbol{\Sigma}(\boldsymbol{\xi})^{-1}\right)\right)}{\partial[\boldsymbol{\xi}]_{q}}\right)\right|_{\boldsymbol{\xi}=\boldsymbol{\xi}_{0}}=\operatorname{tr}\left\{\left.\boldsymbol{\Sigma}\left(\boldsymbol{\xi}_{0}\right)^{-1} \frac{\partial \boldsymbol{\Sigma}(\boldsymbol{\xi})}{\partial \boldsymbol{\xi}}\right|_{\boldsymbol{\xi}=\boldsymbol{\xi}_{0}}+\left.\boldsymbol{\Sigma}\left(\boldsymbol{\xi}_{n}\right) \frac{\partial \boldsymbol{\Sigma}(\boldsymbol{\xi})}{\partial \boldsymbol{\xi}}\right|_{\boldsymbol{\xi}=\boldsymbol{\xi}_{0}}\right\} \\
& \text { and where } \beta_{\text {uncond }}(p, n)=\left.\sum_{q=1}^{2}\left[\boldsymbol{C}_{\mathrm{CRB}}\right]_{p, q}\left(\frac{\partial \kappa_{\text {uncond }}(n, q)}{\partial[\boldsymbol{\xi}]_{q}}\right)\right|_{\boldsymbol{\xi}=\boldsymbol{\xi}_{0}}+\left[\boldsymbol{\xi}_{0}\right]_{p}-\left[\boldsymbol{\xi}_{n}\right]_{p}
\end{aligned}
$$

\section{APPENDIX B}

In this appendix, we rewrite the TTB presented in [14] using the minimization formulation as presented in [16]. The aim is then, to explore the constraints on the bias due to the TTB. In the following, and due to the space limitation, we only consider the case of one unknown parameter denoted $\xi$. The extension of an unknown vector parameter is tedious/long but straightforward and will not be presented in this appendix.

In the case of one unknown parameter $\xi$, one has $\boldsymbol{C}_{\mathrm{TTB}}^{(L, P)}=\boldsymbol{\Gamma}^{H} \boldsymbol{K}^{-1} \boldsymbol{\Gamma}$, where $\boldsymbol{\Gamma}=\left[\begin{array}{c}1 \\ \boldsymbol{W} \boldsymbol{\Phi}\end{array}\right]$, in which $\boldsymbol{\Phi}=\left[\begin{array}{c}\xi_{1}-\xi_{0} \\ \vdots \\ \xi_{1}-\xi_{L}\end{array}\right]$ and $\boldsymbol{K}=\left[\begin{array}{cc}\mathbf{F I M} & \boldsymbol{D}^{\ddagger} \boldsymbol{W}^{H} \\ \boldsymbol{W} \boldsymbol{D} & \boldsymbol{W} \boldsymbol{\Psi} \boldsymbol{W}^{H}\end{array}\right]$, in which $\boldsymbol{D}=-\left[\begin{array}{c}\left.\left(\frac{\partial \operatorname{KLD}\left(p\left(\boldsymbol{\chi} ; \xi_{1}\right) \| p(\boldsymbol{\chi} ; \xi)\right)}{\partial \xi}\right)\right|_{\xi=\xi_{0}} \\ \vdots \\ \left.\left(\frac{\partial \operatorname{KLD}\left(p\left(\boldsymbol{\chi} ; \xi_{L}\right) \| p(\boldsymbol{\chi} ; \xi)\right)}{\partial \xi}\right)\right|_{\xi=\xi_{0}}\end{array}\right]$. In one hand and in order to follow the framework of [14], let us rewrite $\boldsymbol{K}$ as $[\boldsymbol{K}]_{m, n}=\int g_{m} g_{n} d \boldsymbol{\chi}$ where, 


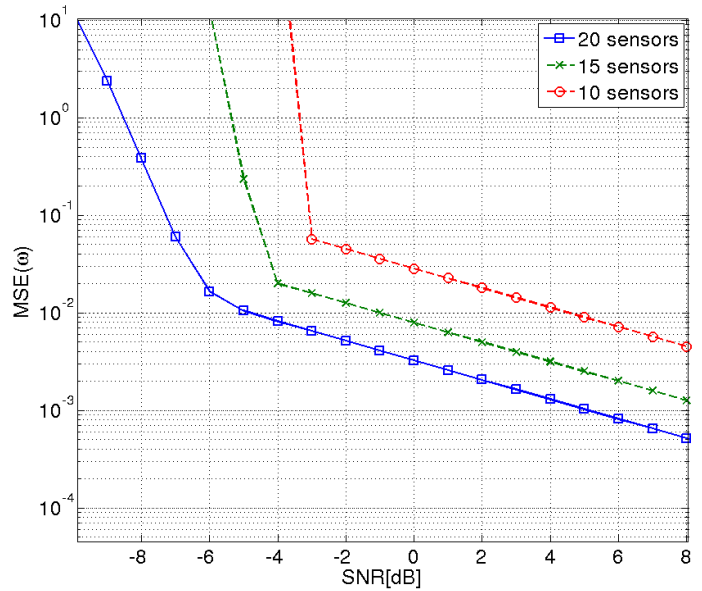

Fig. 6. Deterministic lower bounds on the mean square error w.r.t. $\omega$ for near field source localization, with different number of sensors.

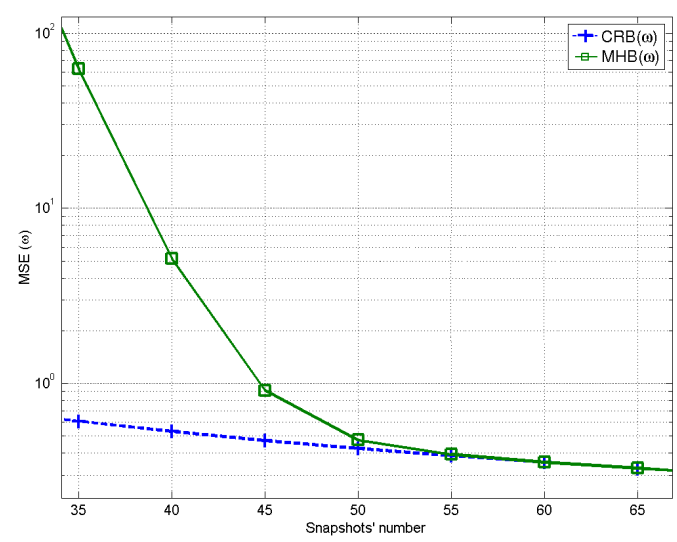

Fig. 7. MHB lower bound on the mean square error w.r.t. $\omega$ for near field source localization.

$$
\begin{aligned}
& g_{1}=\left.\int \frac{\partial \operatorname{Ln} p(\boldsymbol{\chi} ; \xi)}{\partial \xi}\right|_{\xi=\xi_{0}} \sqrt{p\left(\boldsymbol{\chi} ; \xi_{0}\right)} d \boldsymbol{\chi}=\left.\int \frac{\partial p(\boldsymbol{\chi} ; \xi)}{\partial \xi}\right|_{\xi=\xi_{0}} \frac{1}{\sqrt{p\left(\boldsymbol{x} ; \xi_{0}\right)}} d \boldsymbol{\chi}, \text { and } \\
& \quad[\boldsymbol{K}]_{m, 1}=\left.\sum_{n=1}^{L} \int \frac{\partial \operatorname{Ln} p(\boldsymbol{\chi} ; \xi)}{\partial \xi}\right|_{\xi=\xi_{0}} p\left(\boldsymbol{\chi} ; \xi_{n}\right) \exp \left(-j 2 m \pi \frac{n-1}{L}\right) d \boldsymbol{\chi}=\sum_{n=1}^{L} \rho\left(\boldsymbol{\chi}, \xi_{n}\right) \exp \left(-j 2 m \pi \frac{n-1}{L}\right),
\end{aligned}
$$

Note that, $[\boldsymbol{K}]_{m, 1}$ represents the DFT of the sequence $\left\{\rho\left(\boldsymbol{\chi}, \xi_{n}\right)\right\}_{n=1}^{L}$ where $\rho\left(\boldsymbol{\chi}, \xi_{n}\right)=$ $\left.\int \frac{\partial \operatorname{Ln} p(\boldsymbol{\chi} ; \xi)}{\partial \xi}\right|_{\xi=\xi_{0}} p\left(\boldsymbol{\chi} ; \xi_{n}\right) d \boldsymbol{\chi}$. On the other hand, we can rewrite $[\boldsymbol{K}]_{m, 1}$ as follows

$$
[\boldsymbol{K}]_{m, 1}=\left.\sum_{n=1}^{L} \int \frac{\partial \operatorname{Ln} p(\boldsymbol{\chi} ; \xi)}{\partial \xi}\right|_{\xi=\xi_{0}} \sqrt{p\left(\boldsymbol{\chi} ; \xi_{0}\right)} \frac{p\left(\boldsymbol{\chi} ; \xi_{n}\right)}{\sqrt{p\left(\boldsymbol{\chi} ; \xi_{0}\right)}} \exp \left(-j 2 m \pi \frac{n-1}{L}\right) d \boldsymbol{\chi}=\int g_{1} g_{m} d \boldsymbol{\chi}
$$

in which $g_{m}$ represents the DFT of the sequence $\left\{\frac{p\left(\boldsymbol{\chi} ; \xi_{n}\right)}{\sqrt{p\left(\boldsymbol{\chi} ; \xi_{0}\right)}}\right\}_{n=1}^{L}$ given as follows $g_{m}=$ $\sum_{n=1}^{L} \frac{f\left(\boldsymbol{\chi} ; \xi_{n}\right)}{\sqrt{p\left(\boldsymbol{\chi} ; \xi_{0}\right)}} \exp \left(-j 2 m \pi \frac{n-1}{L}\right)$. Since, $\boldsymbol{\Gamma}=\left[\begin{array}{c}1 \\ \boldsymbol{W} \boldsymbol{\Phi}\end{array}\right]$, then the $m^{\text {th }}$ line of the vector $\boldsymbol{W} \boldsymbol{\Phi}$ represents the spectrum frequency for the sequence $\left\{\xi_{n}-\xi_{0}\right\}_{n=1}^{L}$ at the $m^{\text {th }}$ frequency. In the same way, each $g_{m}$ represents the spectrum frequency of the sequence $\left\{\frac{p\left(\boldsymbol{x} ; \xi_{n}\right)}{\sqrt{p\left(\boldsymbol{x} ; \xi_{0}\right)}}\right\}_{n=1}^{L}$ at the $m^{\text {th }}$ frequency.

Finally, the TTB can be viewed as a compression (in the frequency domain) of the Barankin constraints, i.e., the constraint due to the construction of the TTB, are the DFT of a large sequence, this sequence reflects a null bias on several test points.

\section{REFERENCES}

[1] H. Krim and M. Viberg, "Two decades of array signal processing research: the parametric approach," IEEE Signal Processing Mag., vol. 13, no. 4, pp. 67-94, 1996.

[2] Y. D. Huang and M. Barkat, "Near-field multiple source localization by passive sensor array," IEEE Trans. Antennas Propagat., vol. 39, pp. 968-975, 1991. 
[3] N. Yuen and B. Friedlander, "Performance analysis of higher order ESPRIT for localization of near-field sources," IEEE Trans. Signal Processing, vol. 46, pp. 709-719, 1998.

[4] E. Grosicki, K. Abed-Meraim, and Y. Hua, "A weighted linear prediction method for near-field source localization," IEEE Trans. Signal Processing, vol. 53, pp. 3651-3660, 2005.

[5] W. Zhi and M. Chia, "Near-field source localization via symmetric subarrays," IEEE Signal Processing Lett., vol. 14, no. 6, pp. 409-412, 2007.

[6] M. N. El Korso, G. Bouleux, R. Boyer, and S. Marcos, "Sequential estimation of the range and the bearing using the zero-forcing MUSIC approach," in Proc. EUSIPCO, Glasgow, Scotland, Aug. 2009, pp. 1404-1408.

[7] M. N. El Korso, R. Boyer, A. Renaux, and S. Marcos, "Nonmatrix closed-form expressions of the Cramér-Rao bounds for near-field localization parameters," in Proc. of IEEE Int. Conf. Acoust., Speech, Signal Processing, Taipei, Taiwan, 2009.

[8] P. Stoica and A. Nehorai, "MUSIC, maximum likelihood and the Cramér Rao bound," IEEE Trans. Acoust., Speech, Signal Processing, vol. 37, pp. 720-741, May 1989.

[9] — - "MUSIC, maximum likelihood and the Cramér Rao bound: further results and comparisons," IEEE Trans. Acoust., Speech, Signal Processing, vol. 38, pp. 2140-2150, 1990.

[10] E. L. Lehmann, Theory of Point Estimation. New York: Wiley, 1983.

[11] H. Cramér, Mathematical Methods of Statistics. New York: Princeton University, Press, 1946.

[12] E. Boyer, P. Forster, and P. Larzabal, "Non asymptotic statistical performances of beamforming for deterministic signals," IEEE Signal Processing Lett., vol. 11, no. 1, pp. 20-22, Jan. 2004.

[13] L. Atallah, J. P. Barbot, and P. Larzabal, "SNR threshold indicator in data aided frequency synchronization," IEEE Signal Processing Lett., vol. 11, pp. 652-654, Aug. 2004.

[14] K. Todros and J. Tabrikian, "General classes of performance lower bounds for parameter estimation Part I: Non-bayesian bounds for unbiased estimators," IEEE Trans. Inform. Theory, vol. 56, pp. 5045-5063, Oct. 2010.

[15] F. E. Glave, “A new look at the Barankin lower bound," IEEE Trans. Inform. Theory, vol. 18, no. 3, pp. 349-356, May 1972.

[16] P. Forster and P. Larzabal, "On lower bounds for deterministic parameter estimation," in Proc. of IEEE Int. Conf. Acoust., Speech, Signal Processing, Orlando, Fl, 2002.

[17] E. Chaumette, J. Galy, A. Quinlan, and P. Larzabal, "A new Barankin bound approximation for the prediction of the threshold region performance of maximum likelihood estimators," IEEE Trans. Signal Processing, vol. 56, no. 11, pp. 5319-5333, Nov. 2008.

[18] E. W. Barankin, "Locally best unbiased estimates," Ann. Math. Stat., vol. 20, pp. 477-501, 1949.

[19] R. J. Kozick and B. M. Sadler, "Source localization with distributed sensor arrays and partial spatial coherence," IEEE Trans. Signal Processing, vol. 52, pp. 601-616, Mar. 2004.

[20] R. J. McAulay and L. P. Seidman, "A useful form of the Barankin lower bound and its application to ppm threshold analysis," IEEE Trans. Inform. Theory, vol. 15, pp. 273-279, Mar. 1969.

[21] J. M. Hammersley, “On estimating restricted parametrers," J. R. Soc. Ser. B, vol. 12, pp. 192-240, 1950.

[22] L. Atallah, J. P. Barbot, and P. Larzabal, "From Chapman Robbins bound towards Barankin bound in threshold behaviour prediction," Electronic Letters, vol. 40, pp. 279-280, Feb. 2004.

[23] R. J. McAulay and E. M. Hofstetter, "Barankin bounds on parameter estimation," IEEE Trans. Inform. Theory, vol. 17, pp. 669-676, Nov. 1971.

[24] P. Stoica and A. Nehorai, "Performances study of conditional and unconditional direction of arrival estimation," IEEE Trans. Acoust., Speech, Signal Processing, vol. 38, pp. 1783-1795, Oct. 1990.

[25] P. Stoica and R. Moses, Spectral Analysis of Signals. NJ: Prentice Hall, 2005.

[26] K. B. Petersen and M. S. Pedersen, “The matrix cookbook," available at http://matrixcookbook.com, ver. nov. 14, 2008. 